Check for updates

Cite this: Mater. Adv., 2021, 2, 4058

Received 1st April 2021, Accepted 3rd May 2021

DOI: 10.1039/d1ma00301a

rsc.li/materials-advances

\title{
Rare earth free bright and persistent white light emitting zinc gallo-germanate nanosheets: technological advancement to fibers with enhanced quantum efficiency $\dagger$
}

\author{
Bhupendra B. Srivastava, (D)*a Santosh K. Gupta, (D) bc Raul Barbosa, ${ }^{d}$ \\ Alexa Villarreal, ${ }^{d}$ Karen Lozano*d and Yuanbing Mao (D)*e
}

\begin{abstract}
Recent materials science and engineering research focused on defects, dopants, hosts, and morphological structures has resulted in novel cost efficient and sustainable phases with extraordinary properties and performance. Contributing in this direction we have designed dopant and rare earth free white lightemitting zincgallogermanate (ZGG) phosphors in nanosheet (NS) morphology. These ZGG NSs with interlayer and interfacial defects display bright white photoluminescence $(\mathrm{PL})$ with significant quantum yield (QY). Thermal treatment of the as-synthesized ZGG NSs at $750{ }^{\circ} \mathrm{C}$ does not degrade their sheet-like morphology while resulting in long persistent luminescence (PerL) with a duration of approximately one hour. Furthermore, to improve the commercial viability of the as-synthesized ZGG NSs, we have assembled them as fine fibers of polyvinyl alcohol (PVA) using Forcespinning ${ }^{\circledR}$ technology. The ZGG-PVA fibers displayed efficient white PL with increased quantum yield compared to the as-synthesized ZGG NSs. We believe this technological evolution, transitioning from bulk ZGG to ZGG nanosheets, will lead to dopant-free/rare-earth-free persistent white light emission and an enhancement in QY. This technology will be a boon to the optoelectronic and lighting industries, and will benefit commercial applications in smart textiles, energy efficient lighting, night vision, anti-counterfeiting, traffic signals, and security, among other potential uses.
\end{abstract}

\section{Introduction}

Optical materials which display bright emission and exhibit persistent luminescence (PerL) have shown tremendous potential as advanced materials to be used in many aspects of daily human life. ${ }^{1-11}$ PerL is the emission of light which persists for an extended period of time after the cessation of excitation whose intensity and duration depend on trap density and trap depth.

Since the first $\mathrm{SrAl}_{2} \mathrm{O}_{4}: \mathrm{Eu}^{2+}, \mathrm{Dy}^{3+}$ green light-emitting PerL material was synthesized by Matsuzawa et al., the field has rapidly grown within the last decade. ${ }^{12}$ The commercial blue and red PerL phosphors are $\mathrm{CaAl}_{2} \mathrm{O}_{4}: \mathrm{Eu}^{2+}, \mathrm{Nd}^{3+}$ and $\mathrm{Y}_{2} \mathrm{O}_{2} \mathrm{~S}: \mathrm{Eu}^{3+}$,

\footnotetext{
${ }^{a}$ Department of Chemistry, University of Texas Rio Grande Valley, 1201 West University Drive, Edinburg, Texas 78539, USA.

E-mail: bhupendra.srivastava@utrgv.edu

${ }^{b}$ Radiochemistry Division, Bhabha Atomic Research Centre, Trombay, Mumbai-400085, India

${ }^{c}$ Homi Bhabha National Institute, Anushaktinagar, Mumbai, 400094, India

${ }^{d}$ Department of Mechanical Engineering, University of Texas Rio Grande Valley, Edinburg, TX, 78539, USA. E-mail: karen.lozano@utrgv.edu

${ }^{e}$ Department of Chemistry, Illinois Institute of Technology, 3101 South Dearborn Street, Chicago, IL, 60616, USA. E-mail: ymao17@iit.edu

$\dagger$ Electronic supplementary information (ESI) available. See DOI: 10.1039/d1ma00301a
}

$\mathrm{Mg}^{2+}, \mathrm{Ti}^{4+} \cdot{ }^{13}$ Recently however, scientists have been facing several issues in designing cheap luminescent materials owing to overdependence on rare earth ions. There are several other issues associated with rare earth ions such as the global dominance of China, high cost production as well as safety concerns related to their mining. ${ }^{14,15}$ Though still at a very early stage, people around the world have started looking into the replacement of rare earth doped phosphors though there is still a long way to go. Rare earth free phosphors (REFP) are one such class of materials which are receiving extensive attention.

Various inorganic persistent luminescent materials that emit in different regions of spectra have been developed and commercialized. These materials have shown excellent emission intensity, long persistence in emission and stability. Visible light emitting PerL materials are widely used in night vision, interior decorations, displays, and smart textiles as well as in emerging technologies such as anti-counterfeiting, optical recording and bioimaging. . $^{6,9,10,16-21}$ One of the major challenges in bioimaging is large auto-fluorescence background due to tissue excitation in upconversion (UC) and downconversion (DC) applications. The best way to resolve this issue is to separate excitation and emission, which is possible with PerL. ${ }^{9}$ 
Pioneering work in this area was demonstrated by Pan et al. by synthesizing bulk $\mathrm{Zn}_{3} \mathrm{Ge}_{2} \mathrm{Ga}_{2} \mathrm{O}_{10}$ : $\mathrm{Cr}$ (zinc gallogermanate doped with chromium, ZGGC) which is reported to exhibit a long-persistent luminescence of up to $360 \mathrm{~h} .{ }^{4}$ In fact, nanostructured $\mathrm{ZnGa}_{2} \mathrm{O}_{4}: \mathrm{Cr}^{3+}$ and ZGGC are considered the best Near Infra-Red (NIR) PerL materials among this class of inorganic materials. $^{1,22}$ The onward ZGGC as NIR PerL phosphor (mostly nanosized) has garnered enormous attention in the optical research community for several applications. These applications include contrast nanoprobes, cell imaging, night vision surveillance, drug delivery, photodynamic therapy, and latent fingerprint examination.

Most of the research is focused on the exploration of $\mathrm{Zn}_{3} \mathrm{Ga}_{2} \mathrm{Ge}_{2} \mathrm{O}_{10}$ (ZGG) doped with $\mathrm{Mn}^{2+}, \mathrm{Bi}^{3+}$, and $\mathrm{Cr}^{3+}$ for their PL and/or PerL properties owing to its low synthesis temperature, high stability, excellent bio-compatibility, non-toxicity, and moderate conductivity. ${ }^{1,4,18,22-36}$ However, there are very few reports on intrinsic self-activated bluish white emission in bulk/microcrystalline ZGG. ${ }^{29}$ Although Li et al. ${ }^{18}$ have demonstrated the visible PL in ZGG nanoparticles, their emission zone lies more in the green region which lacks commercial usage for day to day activity compared to white light emitting materials.

$\mathrm{Mn}^{2+}, \mathrm{Bi}^{3+}$, and $\mathrm{Cr}^{3+}$ doped ZGG is expected to yield a broad band profile and shorter excited state lifetime owing to involvement of d-electrons. Apart from that, doping can also lead to lattice strain and charge-compensating defects if size and charge mismatch occurs, which affects the emission output, quantum yield (QY) and the afterglow duration of PerL optical materials by providing non-radiative pathways. There have been reports on co-doping $\mathrm{Pr}^{3+}, \mathrm{Ca}^{2+}, \mathrm{Bi}^{3+}$, and $\mathrm{Gd}^{3+}$ ions in ZGG nanocrystals to improve PerL and afterglow duration. ${ }^{1,23,34,37}$ There are a few reports on triply co-doped ZGG with $\mathrm{Cr}^{3+}, \mathrm{Er}^{3+}$ and $\mathrm{Yb}^{3+}$ ions which is further expected to induce high strain in the lattice. ${ }^{27,28,38,39}$ Wang et al. have reported sodium ZGG as blue emitting phosphor whose color can be enhanced by increasing the $\mathrm{Ga}^{3+}$ concentration and can be turned green by doping $\mathrm{Tb}^{3+}$ ions. $^{32}$

The research pertaining to dopant and rare earth free ZGG and its exploitation as PL and PerL phosphors is extremely limited and not well explored, barring very few reports. Ren et al. have explored bluish-white emission in bulk samples of $\mathrm{Zn}_{1+x} \mathrm{Ga}_{2-2 x} \mathrm{Ge}_{x} \mathrm{O}_{4}$ whereas, in this article, for the first time, white light emission from nanosheets (NSs) of ZGG is reported and PL emission further reproduced in PVA fine fibers. ${ }^{29}$

Though the importance of NIR PL and PerL coming from ZGGC or other doped ZGG cannot be ruled out owing to several advantages and applications as mentioned above, pure white light has its own advantages. White light is considered the most important light invention for mankind owing to its day-to-day applicability in houses, the automotive industry, and offices. ${ }^{40-42}$ Moreover, if one can design persistent white light materials, it can be a boon to people who are devoid of electricity in rural areas. Suitable white PerL materials, once excited with photons for a short duration, either by LED or sunlight, can keep glowing for a longer duration, or can be used as luminous lamps. Commercial white light (YAG: $\mathrm{Ce}^{3+}$ pumped by blue LED) requires cerium ion as one of the components which, being a rare-earth metal, increases the cost of the materials. Herein, we have synthesized dopant and rare earth free bright white lightemitting ZGG with bright hue, high intensity, and longer afterglow performance. Only white PerL phosphors, which are rare earth doped bulk materials, have been reported in recent years. ${ }^{17,20,21,43-46}$ Hence they have several limitations such as increased cost, cumbersome synthesis, strained lattice, poor biocompatibility, and low water dispersibility.

Moreover, there is seldom any report on ZGG NSs and such morphology can offer diverse applications in catalysis, sensors, and surface enhanced properties apart from displaying excellent optical properties. ${ }^{47-49}$ ZGG is a solid solution of cubic spinel $\mathrm{ZnGa}_{2} \mathrm{O}_{4}$ and pseudocubic inverse $\mathrm{Zn}_{2} \mathrm{GeO}_{4}$, and it is considered an excellent photocatalyst and optical material. ${ }^{29,50}$ The optical properties of undoped and doped $\mathrm{ZnGa}_{2} \mathrm{O}_{4}$ and $\mathrm{Zn}_{2} \mathrm{GeO}_{4}$ have been explored extensively by our group for UC, DC and PerL phosphors. $^{6-8,16,19}$

Powder phosphor particles suffered from poor water dispersibility, non-flexibility, poor mechanical strength, poor adhesion to film and fibers, lower loading, and biocompatibility issues, which restrict their applications on a large scale. ${ }^{16,51}$ Scientists have found these problems to be a major issue in the scalability and commercial viability of ZGG based PL and PerL phosphors. There have been few reports lately wherein ZGGC nanoparticles have been encapsulated inside organic frameworks, synthesized as glasses, and surface functionalized. ${ }^{22,36,52}$ Recently we used red and green emitting $\mathrm{ZnGa}_{2} \mathrm{O}_{4}: \mathrm{Cr}^{3+}$ nanoparticles and $\mathrm{Zn}_{2} \mathrm{GeO}_{4}: \mathrm{Mn}^{2+}$ nanorod encapsulated PVA polymer for red and green emitting fibers. ${ }^{16}$ But we could only achieve PerL for a very short time $(\sim 500 \mathrm{~s})$ in green emitting fiber and failed to achieve any red PerL. Moreover, red and green light can't produce the same level of brightness as white light can for household and office lighting. In this work, we have resolved these issues by incorporating the designed bright and persistent white light emitting ZGG NSs into polymeric fibers using Forcespinning ${ }^{\circledR}$ technology. The development of a nanofiber membrane provides enhanced handling capability to further increase the promising potential applications such as the opportunity to provide light to rural areas. Novel aspects have been examined in this work, including the synthesis of dopant-free and cost-efficient ZGG in a twodimensional NS structure, which depicts intense and longer white PL and PerL, and the encapsulation within the fibers. White light-emitting fibers with bright and persistent luminescence are expected to offer several advantages to the security, rural electrification and apparel industries.

\section{Experimental}

\subsection{Materials}

Zinc nitrate hexahydrate $\left(\mathrm{Zn}\left(\mathrm{NO}_{3}\right)_{2} \cdot 6 \mathrm{H}_{2} \mathrm{O}, 98 \%\right)$, gallium nitrate hydrate $\left(\mathrm{Ga}\left(\mathrm{NO}_{3}\right)_{3} \cdot x \mathrm{H}_{2} \mathrm{O}, 99.9 \%\right)$, ammonium hydroxide solution (28.0-30.0\% $\mathrm{NH}_{3}$ basis), and germanium oxide $\left(\mathrm{GeO}_{2}, 99.99 \%\right)$ were purchased from Sigma Aldrich. Ethyl alcohol (technical grade) was purchased from Fisher Scientific. Poly(vinyl alcohol) 
(molecular 96\% hydrolyzed) was purchased from Kuraray. Deionized water was produced from a Smart2Pure water purification system. All the chemicals were of analytical grade reagents and used directly without further purification in this work.

\subsection{Synthesis of ZGG NSs}

Synthesis started by using $3.0 \mathrm{mmol}$ of $\mathrm{Zn}\left(\mathrm{NO}_{3}\right)_{2}$ with $2.0 \mathrm{mmol}$ of $\mathrm{GeO}_{2}$ and $2.0 \mathrm{mmol}$ of $\mathrm{Ga}\left(\mathrm{NO}_{3}\right)_{3} \cdot x \mathrm{H}_{2} \mathrm{O}$ mixed in $30 \mathrm{ml}$ of water. An ammonia solution was added to adjust the $\mathrm{pH}$ until achieving the desired value $(\mathrm{pH} \sim 9-10)$ and then it was stirred for 30 minutes. Then, the solution was transferred to a $45 \mathrm{~mL}$ Teflon-lined stainless-steel autoclave where it was heated at $220{ }^{\circ} \mathrm{C}$ for $10 \mathrm{~h}$. The system was left to cool to room temperature and the resulting ZGG NSs were precipitated out of the synthesis solution with the excess volume of the ethanol-water mixture. The purified NSs were separated by centrifugation and subsequently washed two more times with an ethanol-water mixture. The washed ZGG NSs were dried at $110{ }^{\circ} \mathrm{C}$ in a drying oven overnight to obtain the powder. The as-synthesized ZGG NSs were heat treated at $750{ }^{\circ} \mathrm{C}$ for 5 hours in a furnace to obtain the ZGG-750 NSs emitting persistent white light as demonstrated below.

\subsection{Synthesis of luminescent ZGG-PVA fine fibers}

Polymer solutions using polyvinyl alcohol (PVA) with the as-synthesized ZGG NSs at concentrations of $0.3,0.5$, and $1 \mathrm{wt} \%$ were prepared. The ZGG NSs were mixed and homogenized in $13.5 \mathrm{~g}$ of DI water to produce the desired concentrations. $1.5 \mathrm{~g}$ of PVA was incorporated to the aqueous solutions of the ZGG NSs and placed in an oil bath at $75{ }^{\circ} \mathrm{C}$ where they were continuously stirred for 1 hour. Afterward, the solution was left on a stirring plate to stir overnight at room temperature.

The fibers were developed via a Forcespinning ${ }^{\circledR}$ process which uses centrifugal forces to produce fibers from a wide range of polymeric solutions. Two $\mathrm{mL}$ of the prepared solution were fed into the spinneret of a Cyclone ${ }^{\mathrm{TM}} \mathrm{L}-1000 \mathrm{M}$, purchased from FibeRio Technology Corporation (McAllen, USA). The spinneret was then spun at a velocity between 5000 and $7000 \mathrm{rpm}$ which allowed the solution to exit through both ends of the spinneret through the 30-gauge needles. The solution was then elongated onto equally distanced vertical pillars creating fine fibers, which were then collected after each cycle. The fibers were laid on top of one another in a uniformed way to ensure a fine fiber mat.

\subsection{Instrumentation}

Powder X-ray diffraction (XRD) patterns of the ZGG NSs were taken with a Bruker D8 ADVANCE X-ray diffractometer with $\mathrm{Cu} \mathrm{K}_{\alpha 1}$ radiation $(\lambda=0.15406 \mathrm{~nm})$. XRD data were collected by utilizing a scanning mode of $2 \theta$ ranging from $15^{\circ}$ to $80^{\circ}$ with a scanning step size of $0.04^{\circ}$ and a scanning rate of $2.0^{\circ} \mathrm{min}^{-1}$. Transmission electron microscopy (TEM) and high resolution TEM (HRTEM) images were recorded using a Hitachi HF 3300 TEM/STEM system. PL emission and excitation spectra were recorded using an Edinburgh Instrument FLS 980 fluorimeter system with a steady state xenon lamp source. Morphological analysis of the produced fine fibers was carried out using a scanning electron microscope (SEM) (Sigma VP, Carl Zeiss, Jena, Germany).
Persistent luminescence was also recorded on an Edinburgh Instrument FLS 980 fluorometer system in the kinetic scan mode; the ZGG-750 NSs were excited for 5 minutes and then the decay was measured. A $150 \mathrm{~mm}$ BenFlect coated integrating sphere was used to determine the absolute quantum yield (QY) of the NPs. The spectral sensitivity of the spectrometer and the sphere was modified using a calibrated lamp for spectral light throughput.

\section{Results and discussion}

\subsection{X-ray diffraction}

Fig. 1a shows the XRD patterns of the as-synthesized ZGG NSs and the heat treated ZGG-750 NSs along with the $\mathrm{ZnGa}_{2} \mathrm{O}_{4}$ (ZGO) and $\mathrm{Zn}_{2} \mathrm{GeO}_{4}(\mathrm{ZGeO})$ nanostructures that serve as a comparison. Since ZGG is a solid solution of ZGO and ZGeO, no data for it is available in the crystal structure database. ZGG is a solid solution of ZGO (JCPDS No. 38-1240) and ZGeO (JCPDS No. 25-1018), which are both cubic. ${ }^{33}$ The as-synthesized ZGG NCs discussed in this work were prepared with a $\mathrm{Ga}\left(\mathrm{NO}_{3}\right)_{3} \cdot x \mathrm{H}_{2} \mathrm{O}$ to $\mathrm{GeO}_{2}$ ratio of 0.67 with a formula of $\mathrm{Zn}_{1.75} \mathrm{Ga}_{0.5} \mathrm{Ge}_{0.75} \mathrm{O}_{4}$ and are expected to consist of $\mathrm{Ge}_{\mathrm{Ga}}^{\bullet}$ and $\mathrm{Zn}_{\mathrm{Ga}}^{\prime}$ antisite defects. These act as trap centers in the ZGG-750 NSs and are one of the main reasons for their PerL as demonstrated below. XRD patterns of the ZGG synthesized with different $\mathrm{Ga}\left(\mathrm{NO}_{3}\right)_{3} \cdot x \mathrm{H}_{2} \mathrm{O}$ to $\mathrm{GeO}_{2}$ ratios $(0.67,1.0$ and 1.5) have also been shown in Fig. S1 (ESI $\dagger$ ).

XRD patterns (Fig. S2, ESI $\dagger$ ) of the hydrothermally synthesized ZGG NSs from the $\mathrm{Ga}\left(\mathrm{NO}_{3}\right)_{3} \cdot x \mathrm{H}_{2} \mathrm{O}$ to $\mathrm{GeO}_{2}$ ratios of 1.0 and 1.5 and the as-synthesized ZGG NSs after washing with a $0.1 \mathrm{M} \mathrm{NaOH}$ solution at room temperature for 1 hour to dissolve possible unreacted $\mathrm{GeO}_{2}$. These results confirmed that there was no free standing $\mathrm{GeO}_{2}$ as no difference was observed from the ZGG NSs before and after the base treatment.

\subsection{FESEM and TEM morphostructural investigation}

A FESEM image (Fig. S3a, ESI $\dagger$ ) shows a highly monodispersed nanodisk/sheet formation with an average diameter in the range of $70 \pm 5 \mathrm{~nm}$. It can clearly be observed that all ZGG particles have sheet morphology with different dimensions. The elemental analysis was carried out using FESEM which clearly shows the presence of $\mathrm{Zn}, \mathrm{Ga}$ and Ge with their corresponding atomic and weight percentage shown in the inset of Fig. S3b (ESI $\dagger$ ). These NSs can also be seen at lower magnifications (Fig. S4, ESI $\dagger$ ).

To further validate the morphology of the ZGG, we carried out HRTEM analysis (Fig. 1b-d). HRTEM clearly shows that hydrothermally synthesized ZGG NSs have 2D sheet morphology with an average size of $70 \pm 5 \mathrm{~nm}$. These NSs are expected to have defects and interfaces which aid in the PerL in the ZGG NSs along with the $\mathrm{Ge}_{\mathrm{Ga}}^{\bullet}$ and $\mathrm{Zn}_{\mathrm{Ga}}^{\prime}$ antisite defects. NSs results in an abundance of defects found on their surface and edges and between layers. Different sized NSs and interlayers were also identified in the HRTEM images, which aids the PerL effect. The HRTEM images of the as-synthesized ZGG NSs show lattice fringes in the NSs (Fig. S5a, ESI $\dagger$ ), with the lattice spacing of $0.31 \mathrm{~nm}$ in addition to fringes due to overlapping of $2 \mathrm{D}$ NSs (Fig. S5b, ESI $\dagger$ ). Moreover, the ZGG-750 NSs retained the 

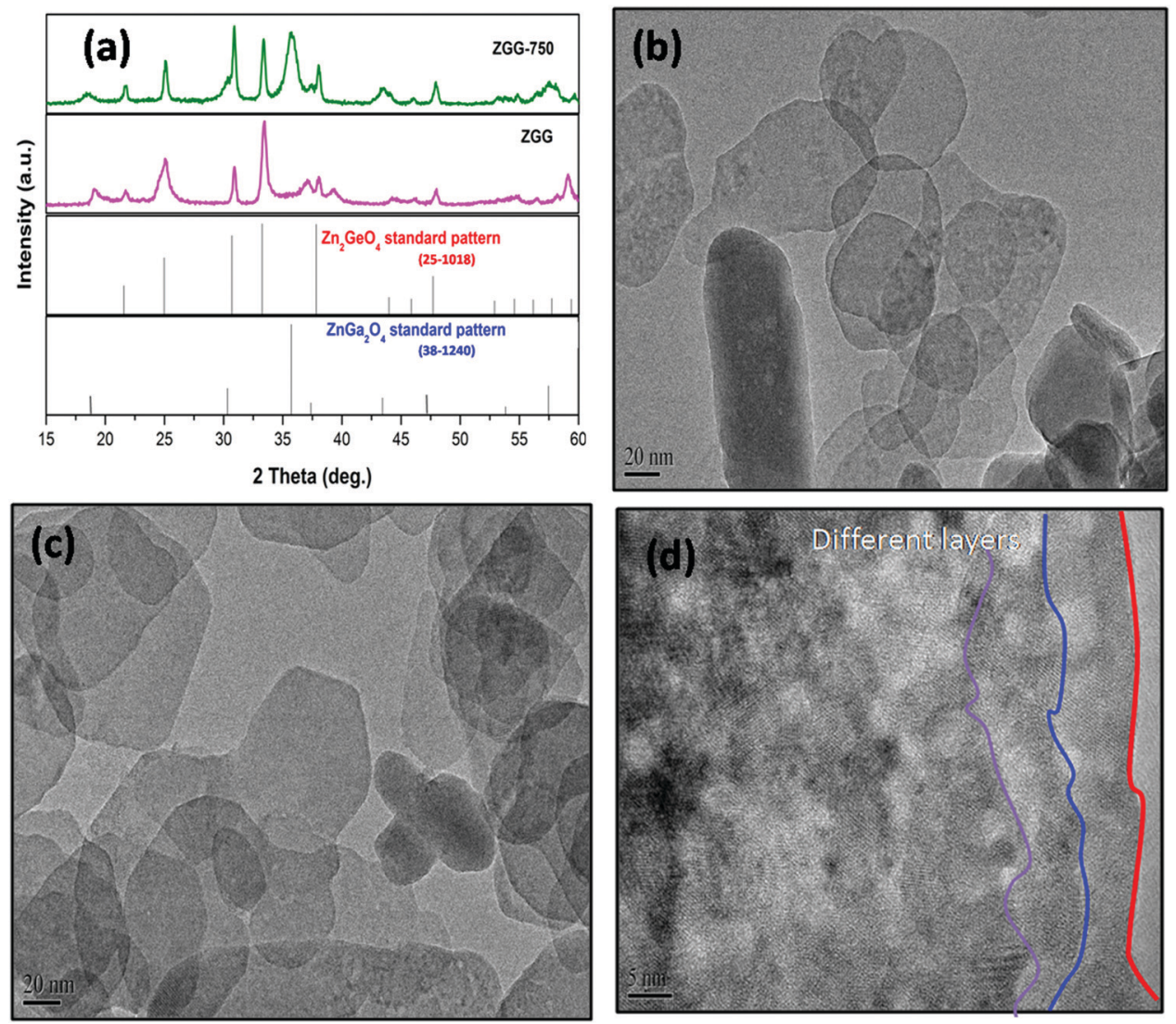

Fig. 1 (a) XRD patterns of the as-synthesized ZGG NSs prepared with the $\mathrm{Ga}\left(\mathrm{NO}_{3}\right)_{3} \cdot \mathrm{XH}_{2} \mathrm{O}$ to a $\mathrm{GeO}_{2}$ precursor ratio of 0.67 and the corresponding ZGG-750 NSs along with standard stick patterns of $\mathrm{ZnGa}_{2} \mathrm{O}_{4}$ and $\mathrm{Zn}_{2} \mathrm{GeO}_{4}$ and (b-d) HRTEM images of the as-synthesized ZGG NSs.

sheet-shape morphology of the as-synthesized ZGG NSs (Fig. S6, ESI $\dagger$ ) but clustered together.

\subsection{Photo and persistence luminescence of ZGG NSs}

The photoluminescence excitation spectra of the as-synthesized ZGG NSs and the ZGG-750 NSs under $515 \mathrm{~nm}$ emission (Fig. 2a) showed a dual feature: (a) an intense broad band $\left(\mathrm{B}_{1}\right)$ peaking around $275 \mathrm{~nm}$ (approximately $4.5 \mathrm{eV}$ ) and (b) a small peak $\left(\mathrm{B}_{2}\right)$ around $320 \mathrm{~nm}$.

The band $\mathrm{B} 1$ is ascribed to the electronic transition band from the valence to the conduction band (CB) of ZGG. The B1 band is located close to the band gap of a material which is known as the host absorption band. The band gap of ZGeO and ZGO is also close to $4.4-4.5 \mathrm{eV}$ and therefore the ZGG NSs are expected to have a band gap in the same region. The second band, B2, arises due to structural defects and defect clusters present in ZGG. The PLE spectrum of the ZGG-750 NSs showed a small red shift of the $\mathrm{B} 2$ band owing to the thermal treatment of the as-synthesized ZGG NSs.

Photoluminescence emission spectra of the as-synthesized ZGG NSs and the ZGG-750 NSs under $275 \mathrm{~nm}$ excitation (Fig. 2b) depicted broad emission profiles spanning $350-700 \mathrm{~nm}$ in the visible region. There was no change in the emission profile or intensity of the as-synthesized ZGG NSs and the ZGO-750 NSs. Such bright white light in a non-activated sample, suggests the presence of several defects in the band gap of the ZGG NSs. As mentioned earlier, the ZGG is a solid solution of ZGO and ZGeO, both of which are also known to exhibit self-activated blue and green emission, respectively. ${ }^{6,53}$

Blue emission from the ZGO is attributed to recombination of $\mathrm{Ga}^{3+}$ ions with electrons trapped in oxygen vacancies under UV illumination. Similarly, the bright green emission in ZGeO is attributed to structural distortion particularly singly ionized oxygen vacancies $\left(\mathrm{V}_{\mathrm{O}}^{\bullet}\right)$. Moreover, the zinc interstitial $\left(\mathrm{Zn}_{\mathrm{i}}^{\bullet}\right) /$ vacancies $\left(\mathrm{V}_{\mathrm{Zn}}^{\prime \prime}\right)$ and germanium vacancies $\left(\mathrm{V}_{\mathrm{Ge}}^{0}\right)$ contribution to the green PL of ZGeO couldn't be ruled out as they are present in abundance. ${ }^{54}$ The donor in ZGeO is singly ionized oxygen vacancies $\left(\mathrm{V}_{\mathrm{O}}^{\bullet}\right) /\left(\mathrm{Zn}_{\mathrm{i}}^{\bullet}\right)$ whereas the acceptor is $\mathrm{V}_{\mathrm{Zn}}^{\prime \prime} /\left(\mathrm{V}_{\mathrm{Ge}}^{0}\right)$ and the donor-acceptor recombination leads to green PL. It is expected that the PL of the ZGG NSs, being the solid solution of ZGO and ZGeO, should share a similar emission mechanism and that is radiative donor-acceptor recombination. The emissive centers in solids will be highly complex. It must recombine $\mathrm{V}_{\mathrm{O}}^{\bullet}$ which acts as a donor in the ZGG NSs with a defect cluster such as $\left(\mathrm{V}_{\mathrm{O}}^{\bullet}-\mathrm{V}_{\mathrm{Ga}}^{\prime \prime \prime}\right)$, $\left(\mathrm{V}_{\mathrm{O}}^{\bullet}-\mathrm{V}_{\mathrm{Ge}}^{0}\right)$ which are the expected acceptor. The color coordinate diagram of the as-synthesized ZGG NSs and the ZGG-750 NSs under $275 \mathrm{~nm}$ excitation is also shown in Fig. 2c (marked by a and $\mathrm{b}$, respectively), which clearly depicts white light emission from both the as-synthesized ZGG NSs and ZGG-750 with a CIE index of $(0.315,0.275)$ and $(0.319,0.278)$ respectively. The details of 

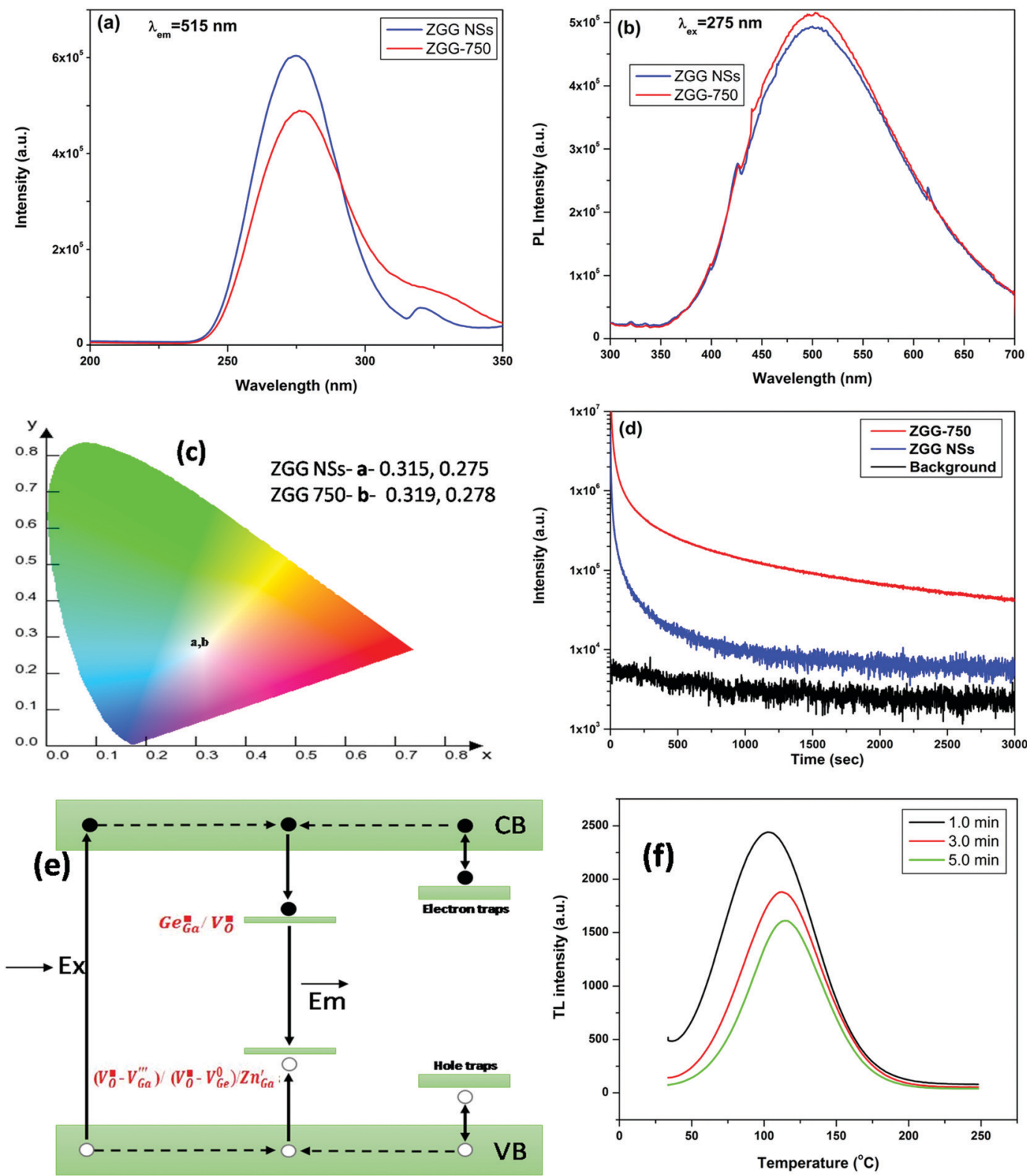

Fig. 2 (a) PLE spectrum, (b) PL spectrum, and (c) color coordinate diagram of the as-synthesized ZGG NSs and the ZGG-750 NSs. (d) PerL decay curves of the as-synthesized ZGG and ZGG-750 NSs. (e) Plausible persistent luminescence mechanism of the ZGG-750 NSs and (f) TL glow curves of the ZGG750 NSs as a function of the irradiation time: $1 \mathrm{~min}, 3 \mathrm{~min}$, and $5 \mathrm{~min}$.

color coordinate calculation are mentioned in the information S1 in the ESI.†

The as-synthesized ZGG NSs display very weak PerL when they are excited by UV light at $256 \mathrm{~nm}$ (Fig. 2d, red curve). On the other hand, the ZGG-750 NSs show a white afterglow for up to $1 \mathrm{~h}$ with a very bright and high-count rate once irradiated with $256 \mathrm{~nm}$ for $5 \mathrm{~min}$ (Fig. 2d, green curve). A video was recorded with a digital camera to demonstrate the persistent white light emission which can be seen with the naked eye for 30 seconds after the UV lamp is switched off (Video S1, ESI $\dagger$ ). We have also carried out still photography with a Samsung Mobile phone and taken a digital image of the light emission from ZGG-750 NSs under a $254 \mathrm{~nm}$ UV lamp at different time intervals, which is shown in Fig. S7 (ESI $\dagger$ ). The mechanism of the long PerL from the ZGG-750 NSs is schematically shown in Fig. $2 \mathrm{e}$.

In the ZGG-750 NSs, $\mathrm{Ge}_{\mathrm{Ga}}^{\cdot} / \mathrm{V}_{\mathrm{O}}^{\bullet}$ (ETs) and $\left(\mathrm{V}_{\mathrm{O}}^{\bullet}-\mathrm{V}_{\mathrm{Ga}}^{\prime \prime \prime}\right) /$ $\left(\mathrm{V}_{\mathrm{O}}^{\bullet}-\mathrm{V}_{\mathrm{Ge}}^{0}\right) / \mathrm{Zn}_{\mathrm{Ga}}^{\prime}$ (HTs) respectively act as electron and hole traps. On irradiating ZGG-750 NSs with $256 \mathrm{~nm}$ light; an abundance of electrons in the conduction band (CB) and holes in the valence bond (VB) are formed. Following excitation, $\mathrm{CB}$ electrons are trapped at ETs and VB holes are trapped at HTs before they can recombine. On thermal activation, recombination of photo-excited holes from HTs and electrons from ETs leads to bright white emission.

The defect traps are at a far off distance in the ZGG host lattice and their recombination becomes a remote possibility at room temperature, as is observed for the as-synthesized 
ZGG NSs (blue curve in Fig. 2d). Thermal treatment of the assynthesized ZGG NSs at $750{ }^{\circ} \mathrm{C}$ enables p-d repulsion between the oxygen $2 \mathrm{p}$ and zinc $3 \mathrm{~d}$ electrons, resulting in aggregation of defect states near the valence band maxima and thereby enhancing the persistent luminescence lifetime of the ZGG-750 NSs. ${ }^{27}$ These holes and electrons trapped in respective defect state traps can be stored until they are not thermally activated for subsequent release to the valence and conduction bands, respectively. PerL in the ZGG-750 NSs originates from the recombination of the released charge carriers at the adjacent vacancy defects acting as luminescence centers. Several defects present on the edges and interfaces of nanosheets also act as trap centers and help in the PerL process. Only a handful of white PerL phosphors have been reported in recent years. ${ }^{17,20,21,43-46}$ The white light from PerL phosphors is composite light and can be obtained by precisely mixing phosphors of different PerL colors in an appropriate ratio. It is hard to ensure phosphorescence color uniformity for different phosphors as it is difficult to achieve consistency in the decay process.

\subsection{Thermoluminescence}

TL glow curves are considered one of the most important measurements to decipher useful information about traps for the exploration of the PerL mechanism. TL measurements on the ZGG-750 NSs after UV irradiation for $1 \mathrm{~min}, 3 \mathrm{~min}$ and 5 min have been carried out (Fig. 2f). The glow curves show that there are peaks located at $102{ }^{\circ} \mathrm{C}, 112{ }^{\circ} \mathrm{C}$, and $116{ }^{\circ} \mathrm{C}$ after irradiation for $1 \mathrm{~min}, 3 \mathrm{~min}$ and $5 \mathrm{~min}$, respectively. This is unlike the ZGG bulk samples where three peaks are present in the TL curves. ${ }^{29}$ As the TL peak is located above room temperature (RT), the ZGG-750 NSs are considered as an excellent material for long lasting PerL. ${ }^{32}$ Uniform singular white persistent emission is again confirmed by the presence of a single peak in the TL glow curves.

Furthermore, irradiation time plays a critical role on the filling of the traps present in the ZGG-750 NSs. The peak intensity decreases monotonically as the irradiation time increases from 1 to $3 \mathrm{~min}$ and then to $5 \mathrm{~min}$ (Fig. 2f), which suggests a gradual filling of the traps of several depths and energies. Time dependence trap filling was ascribed to tunneling of carriers from the trap to the recombination centre. As the irradiation moves to a longer time, TL peak intensity decrease and the peak positions shift to the high-temperature region $\left(102 \rightarrow 112 \rightarrow 116{ }^{\circ} \mathrm{C}\right)$, indicating that different irradiation energies can fill traps with different depths.

Usually, shallow traps are emptied easily even at RT whereas charge carrier in the deep traps stays for longer time and difficult to be emptied at RT. ${ }^{33}$ Based on a singular peak observed in the TL glow curve, the trap is difficult to categorise as either shallow or deep. But we can propose some idea based on the trap depth value which in this case was determined to be $0.87 \mathrm{eV}$ based on the formulation described by Wan et al. ${ }^{55}$ This trap depth is considered decent for long persitent luminescence. The calculated value of trap depth suggests that deep traps are higher in density compared to shallow ones in the ZGG-750 NSs and prominently take part in PerL. ${ }^{36}$ The suitable TL peak is situated slightly above room temperature for excellent persistent luminescence. Therefore, the TL bands centered at $\sim 375-390 \mathrm{~K}$ can be responsible for the white persistent emission.

\subsection{White light emitting ZGG-PVA fibers}

3.5.1. Morphostructural and thermo-physical analysis. FESEM image of the developed ZGG-PVA fibers with $1.0 \mathrm{wt} \%$ loading of the as-synthesized ZGG NSs is shown in Fig. 3a. The inset in this figure shows a digital image of the developed mat. Some of the other FESEM images taken for the ZGG-PVA fibers with $0.3 \mathrm{wt} \%$ and $0.5 \mathrm{wt} \%$ loadings of the as-synthesized ZGG NSs are given in Fig. S8a and b (ESI $\dagger$ ). All of the FESEM images clearly show long, continuous, bead free homogeneous systems. The ZGG-PVA membranes depict individual fibers entangled into each other as typically observed for a non-woven system. ${ }^{16}$ The representative elemental analysis on the ZGG-PVA fibers with $1.0 \mathrm{wt} \%$ loading of the as-synthesized ZGG NSs clearly shows the presence of $\mathrm{Zn}, \mathrm{Ga}, \mathrm{Ge}$ and $\mathrm{O}$ without any impurity (Fig. S9, ESI $\dagger$ ). The peak at $0.277 \mathrm{keV}$ is ascribed to carbon $\mathrm{K} \alpha_{1}$ which may have appeared due to use of carbon tape. There is
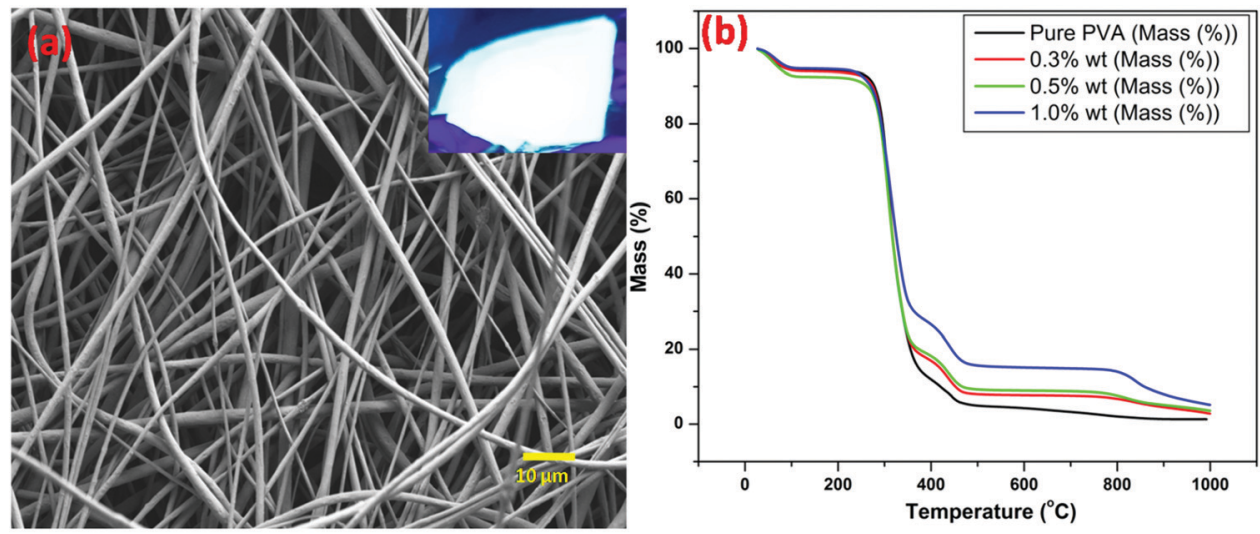

Fig. 3 (a) FESEM image of the ZGG-PVA fine fibers with $1.0 \mathrm{wt} \%$ loading of the as-synthesized ZGG NSs. The inset represents a digital image of the light emission under UV irradiation for the developed nanofiber membrane. (b) TGA curves of the developed ZGG-PVA fine fibers with 0.3 wt\%, 0.5 wt\% and $1.0 \mathrm{wt} \%$ loadings of the as-synthesized ZGG NSs along with the unloaded PVA fibers. 
another peak at around $1.75-1.85 \mathrm{keV}$ which is an internal fluorescence peak appearing due to the X-ray line of silicon $\left(K \alpha_{2}\right.$ and $\left.K \beta_{1}\right)$ owing to the Si dead layer of the Si-Li detector. TGA analysis was also performed on the ZGG-PVA fine fibers with different loadings (i.e., $0.3 \mathrm{wt} \%, 0.5 \mathrm{wt} \%$, and $1.0 \mathrm{wt} \%$ of the as-synthesized ZGG NSs) in order to understand the thermal stability after the encapsulation of the nanosheets. The respective thermograms are shown in Fig. 3b. The TGA thermograph of the PVA polymeric fibers imbedded with as-synthesized ZGG NSs depicts a superposition of two sigmoid functions representing the thermal degradation of both the polymer and fillers. The transition occurring before $200{ }^{\circ} \mathrm{C}$ can be attributed to evaporation/ volatilization of bound/free water molecules in the fibers. ${ }^{56,57}$ The second weight loss observed between $200{ }^{\circ} \mathrm{C}$ and $400{ }^{\circ} \mathrm{C}$, follows the single asymmetric sigmoid observed in PVA systems. Even though the addition of the as-synthesized ZGG NSs resulted in a small shift of the PVA degradation, it did not affect the overall decomposition of the developed polymer
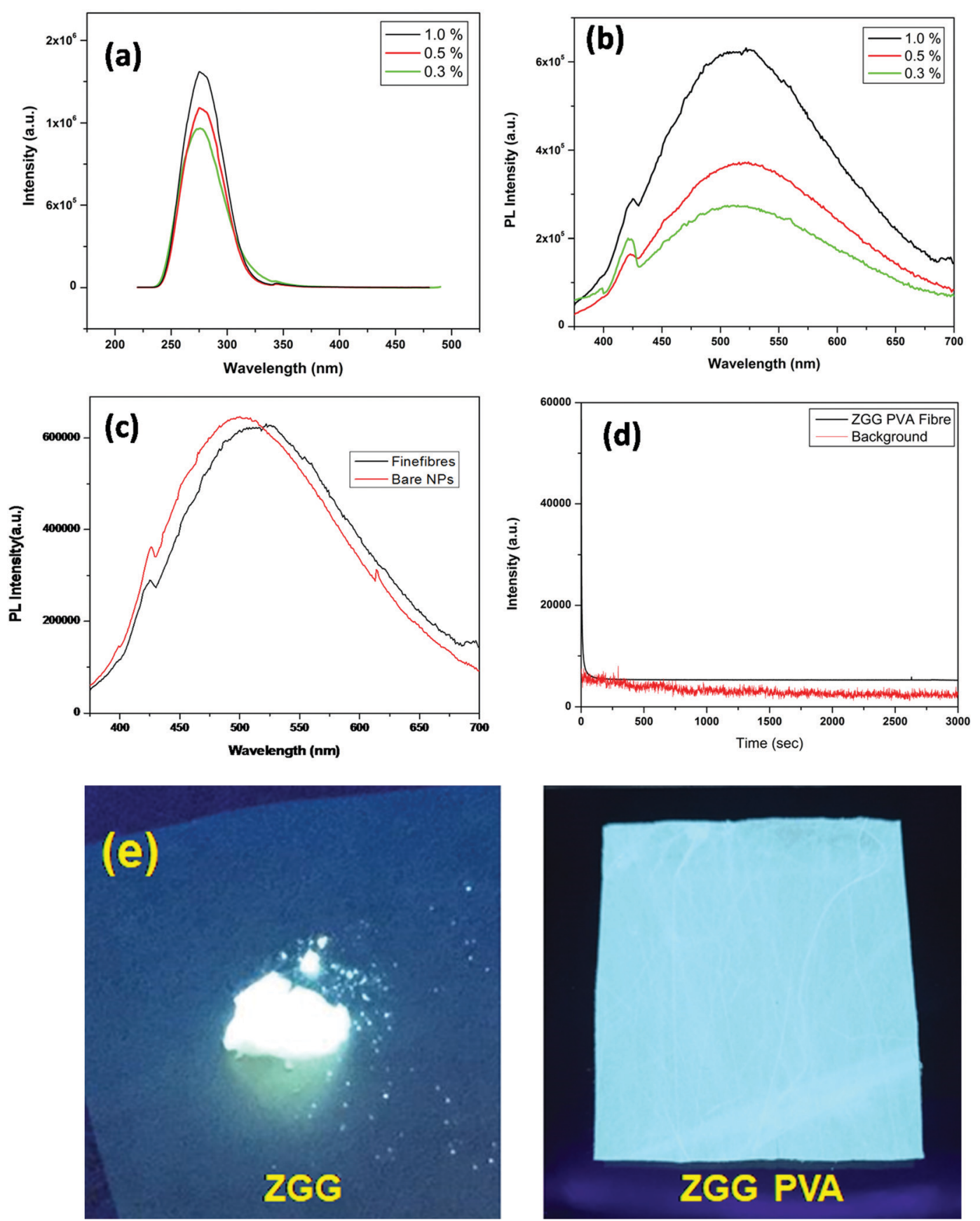

Fig. 4 (a) PLE and (b) PL emission spectra of the ZGG-PVA fine fibers with 0.3 wt\%, 0.5 wt\%, and 1.0 wt\% loadings of the as-synthesized ZGG NSs. (c) Comparative emission spectra of the as-synthesized ZGG NSs and the ZGG-PVA fine fibers. (d) PerL decay curve of the ZGG (1.0 wt\%)-PVA fibers. (e) Digital images of the as-synthesized ZGG NSs and the ZGG (1.0 wt\%)-PVA fibers under UV lamp excitation at $254 \mathrm{~nm}$. 
doped system. The $0.3 \mathrm{wt} \%, 0.5 \mathrm{wt} \%$, and $1.0 \mathrm{wt} \%$ fillers added to the aqueous solutions represent $3.2 \mathrm{wt} \%, 4.8 \mathrm{wt} \%$, and $10.2 \mathrm{wt} \%$, respectively, when water is not accounted for. A residual mass of $4.9 \mathrm{wt} \%, 8.0 \mathrm{wt} \%, 9.3 \mathrm{wt} \%$, and $15.6 \mathrm{wt} \%$ for the pure PVA fibers, $0.3 \mathrm{wt} \%, 0.5 \mathrm{wt} \%$, and $1.0 \mathrm{wt} \%$ samples can be seen in the resulting graphs. The increased residue mass of the doped PVA fiber samples is associated with residual carbon and interaction between the nanosheets and the PVA molecules. The filler creates confinements in parts of the PVA molecules which prevent them from fully degrading at such temperatures.

3.5.2. Photoluminescence spectroscopy of ZGG-PVA fine fibers. We successfully reproduce the excitation (Fig. 4a) and emission band (Fig. 4b) of the as-synthesized ZGG-NS after encapsulation in PVA fibers. Moreover, there is no change in the spectral profile and the white emission band position. The relative PL emission intensity of the ZGG-PVA fine fibers is lower than the as-synthesized ZGG NSs owing to some quenching of defect states (Fig. 4c). A red-shift of the PL peak from the forcespun ZGG-PVA fibers compared to the as-synthesized ZGG NSs is also observed. Furthermore, it could be seen that the polymeric fibers can easily accommodate up to $1.0 \mathrm{wt} \%$ of the as-synthesized ZGG NSs without undergoing formation of aggregate/concentration induced quenching. There is a monotonic increase in both excitation and emission intensities until $1.0 \mathrm{wt} \%$. After that, there is quenching in both intensities. PL quantum yield (PLQY) was also measured for the as-synthesized ZGG NSs and the ZGG-PVA fine fibers (1.0 wt\% loading). Due to the good dispersion of the as-synthesized ZGG NSs inside the PVA polymer matrix, an enhancement in PLQY from $11.7 \%$ of the pristine as-synthesized ZGG NSs to $15.4 \%$ of the ZGG-PVA fibers was obtained. This highlights the advantage of moving from nanopowder to NSs in terms of persistent luminesce, intensity, and duration. Furthermore, the usage of fine fibers helps to achieve a higher quantum yield which is essential for any commercial applications and optoelectronic devices. Uniform and bright white emission further confirms the homogeneous distribution of the ZGG NSs inside the PVA polymeric fine fibers. The bright white emission shown by the ZGG-PVA fine fibers and the ZGG NSs under UV photon excitation can be seen from the digital image shown in Fig. 4e. We believe such fibers can be a boon to the apparel industry in making textiles which can be used for several applications like night vision surveillance, security patrolling, anti-counterfeiting, and several other applications. The idea behind this is to use this fabric in making unique patterns on clothes such as shorts, t-shirts, gloves, fabric or any other textile materials which will emit and sustain light just by exposure to UV light. This can substantially help reduce textile fraud.

The only issue here is that the ZGG-PVA fibers failed to give persistent luminesce as the as-synthesized ZGG NSs were embedded (Fig. 4d). We could not load the ZGG-750 NSs into the PVA fiber precursor owing to the lack of hydroxyl group on their surface as these persistent NSs were formed after thermal annealing of the as-synthesized ZGG NSs at $750{ }^{\circ} \mathrm{C}$ which eliminated the surface hydroxyl groups on these NSs. Still, we believe that we will take care of this in our future work by functionalizing the ZGG NSs to see whether we can incorporate some sort of persistency in our ZGG-PVA fibers.

\section{Conclusions}

In this work, pure ZGG nanosheets as a solid solution of $\mathrm{ZnGa}_{2} \mathrm{O}_{4}$ and $\mathrm{Zn}_{2} \mathrm{GeO}_{4}$ have been synthesized using a hydrothermal method and subjected to characterization using XRD, EDX, FESEM, and HRTEM. Photoluminescence spectroscopy indicated a very bright white light emission from these novel dopant-free ZGG NSs owing to the presence of several defects and further aided by the interfacial and interlayer defects of nanosheets. Interestingly, with the thermal treatment of these as-synthesized ZGG NSs at $750{ }^{\circ} \mathrm{C}$, we could see long persistent luminescence lasting up to one hour. The same has been ascribed to the enhancement in trap densities such as vacancies, antisites, and interstitials. Thermoluminescence measurement on the thermally treated ZGG-750NSs suggested the predominant involvement of deep traps. To further harness the potential of this great material, the as-synthesized ZGG NSs have been encapsulated inside PVA using Forcespinning ${ }^{(\mathbb{R})}$ technology. The resulting ZGG-PVA fibers exhibited fine fiber morphology and a smooth texture without the formation of beads. The thermal stability of the fibers does not deteriorate on nanosheet loading, and fibers can easily accommodate up to $1.0 \mathrm{wt} \%$ of the assynthesized ZGG NSs. Photoluminescence measurements of the ZGG-PVA fibers could easily reproduce the same excitation and emission profiles with almost equivalent intensity and peak profiles. The fibers could display very bright white light emission with enhanced quantum yield compared to the as-synthesized ZGG NSs. This has been ascribed to the better dispersion of the NSs inside the polymeric fibers. This study has made great advances in the development of cost effective and sustainable dopant-free white light-emitting phosphors with long persistent luminescence and improved quantum yields as fibers. This technological advancement from bulk ZGG to the as-synthesized ZGG NSs to the thermally treated ZGG-750 NSs and the ZGGPVA fibers is a great stride in achieving high optoelectronic performance within the concept of defects and materials engineering.

\section{Conflicts of interest}

The authors have no conflicts of interest to declare.

\section{Acknowledgements}

YM would like to thank the financial support from the National Science Foundation under CHE (award \#1952803and 1710160) and the IIT start-up funds. KL, YM, RB, AV, and BS gratefully acknowledge support by the NSF PREM DMR-1523577. SKG thanks the United States-India Education Foundation (USIEF) and the Institute of International Education (IIE) for his Fulbright Nehru Postdoctoral Fellowship (award\#2268/FNPDR/2017). The authors would like to thank Dr Y. Li (School of Physics and 
Optoelectronic Engineering, Guangdong University of Technology) for the TL measurements.

\section{References}

1 A. Abdukayum, J.-T. Chen, Q. Zhao and X.-P. Yan, Functional near infrared-emitting $\mathrm{Cr}^{3+} / \mathrm{Pr}^{3+}$ co-doped zinc gallogermanate persistent luminescent nanoparticles with superlong afterglow for in vivo targeted bioimaging, J. Am. Chem. Soc., 2013, 135, 14125-14133.

2 L. V. S. França and O. Baffa, Boosted UV emission on the optically and thermally stimulated luminescence of $\mathrm{CaB}_{6} \mathrm{O}_{10}$ : Gd,Ag phosphors excited by X-rays, Appl. Mater. Today, 2020, 21, 100829, DOI: 10.1016/j.apmt.2020.100829.

3 W. Li, et al., White luminescent single-crystalline chlorinated graphene quantum dots, Nanoscale Horiz., 2020, 5, 928-933, DOI: 10.1039/d0nh00053a.

4 Z. Pan, Y.-Y. Lu and F. Liu, Sunlight-activated long-persistent luminescence in the near-infrared from $\mathrm{Cr}^{3+}$-doped zinc gallogermanates, Nat. Mater., 2012, 11, 58-63.

5 G. Potsi, et al., Intrinsic photoluminescence of aminefunctionalized graphene derivatives for bioimaging applications, Appl. Mater. Today, 2019, 17, 112-122, DOI: 10.1016/j.apmt. 2019.08.002.

6 B. B. Srivastava, S. K. Gupta, Y. Li and Y. Mao, Bright persistent green emitting water-dispersible $\mathrm{Zn}_{2} \mathrm{GeO}_{4}: \mathrm{Mn}$ nanorods, Dalton Trans., 2020, 49, 7328-7340, DOI: 10.1039/ dodt00361a.

7 B. B. Srivastava, S. K. Gupta and Y. Mao, Remarkable enhancement of photoluminescence and persistent luminescence of NIR emitting $\mathrm{ZnGa}_{2} \mathrm{O}_{4}: \mathrm{Cr}^{3+}$ nanoparticles, CrystEngComm, 2020, 22, 2491-2501, DOI: 10.1039/d0ce00182a.

8 B. B. Srivastava, S. K. Gupta and Y. Mao, Single red emission from upconverting $\mathrm{ZnGa}_{2} \mathrm{O}_{4}$ : $\mathrm{Yb}$,Er nanoparticles co-doped by $\mathrm{Cr}^{3+}$, J. Mater. Chem. C, 2020, 8, 6370-6379, DOI: 10.1039/ d0tc00411a.

9 H.-X. Tan, T.-Y. Wang, C.-Y. Yu and L.-D. Hu, Crucial breakthrough of functional persistent luminescence materials for biomedical and information technological, Front. Chem., 2019, 7, 387.

10 N. S. M. Viswanath, et al., A new persistent blue-emitting phosphor: Tailoring the trap density for enhancing the persistent time, Appl. Mater. Today, 2020, 18, 100518, DOI: 10.1016/j.apmt.2019.100518.

11 Q. Xu, et al., Hydrochromic full-color MXene quantum dots through hydrogen bonding toward ultrahigh-efficiency white light-emitting diodes, Appl. Mater. Today, 2019, 16, 90-101, DOI: 10.1016/j.apmt.2019.05.001.

12 T. Matsuzawa, Y. Aoki, N. Takeuchi and Y. Murayama, A new long phosphorescent phosphor with high brightness, $\mathrm{SrAl}_{2} \mathrm{O}_{4}: \mathrm{Eu}^{2+}, \mathrm{Dy}^{3+}$, J. Electrochem. Soc., 1996, 143, 2670.

13 D. Poelman, D. V. D. Heggen, J. Du, E. Cosaert and P. F. Smet, Persistent phosphors for the future: Fit for the right application, J. Appl. Phys., 2020, 128, 240903, DOI: 10.1063/ 5.0032972 .
14 S. K. Gupta, et al., Achieving Bright Blue and Red Luminescence in $\mathrm{Ca}_{2} \mathrm{SnO}_{4}$ through Defect and Doping Manipulation, J. Phys. Chem. C, 2020, 124, 16090-16101, DOI: 10.1021/ acs.jpcc.0c03180.

15 R. Gupta, et al., Understanding the Dynamics of $\mathrm{Eu}^{3+}$ Ions in Room-Temperature Ionic Liquids - Electrochemical and Time-Resolved Fluorescence Spectroscopy Studies, Eur. J. Inorg. Chem., 2015, 104-111, DOI: 10.1002/ejic.201402713. 16 R. Barbosa, et al., Bright and Persistent Green and Red Light-emitting Fine Fibers: A Potential Candidate for Smart Textiles, J. Lumin., 2020, 117760, DOI: 10.1016/j.jlumin. 2020.117760.

17 D. Jia, J. Girardi and K. Greenland, Long Persistent White Light Emitting Diode, ECS Trans., 2019, 6, 11-17, DOI: 10.1149/1.2938744.

18 L. Li, F. Pan, P. A. Tanner and K.-L. Wong, Tunable Dual Visible and Near-Infrared Persistent Luminescence in Doped Zinc Gallogermanate Nanoparticles for Simultaneous Photosensitization and Bioimaging, ACS Appl. Nano Mater., 2020, 3, 1961-1971.

19 B. B. Srivastava, A. Kuang and Y. Mao, Persistent luminescent sub-10 nm $\mathrm{Cr}$ doped $\mathrm{ZnGa}_{2} \mathrm{O}_{4}$ nanoparticles by a biphasic synthesis route, Chem. Commun., 2015, 51, 7372-7375, DOI: 10.1039/c5cc00377f.

20 L. Zhang, X. Zhou, H. Zeng, H. Chen and X. Dong, Whitelight long-lasting phosphor $\mathrm{Sr}_{2} \mathrm{SiO}_{4}: \mathrm{Pr}^{3+}$, Mater. Lett., 2008, 62, 2539-2541, DOI: 10.1016/j.matlet.2007.12.043.

21 S. Zhang, et al., White-light long persistent luminescence of $\mathrm{Tb}^{3+}$-doped $\mathrm{Y}_{3} \mathrm{Al}_{2} \mathrm{Ga}_{3} \mathrm{O}_{12}$ phosphor, J. Alloys Compd., 2017, 729, 418-425, DOI: 10.1016/j.jallcom.2017.09.169.

22 Y. Lv, et al., Chromium-doped zinc gallogermanate@ zeolitic imidazolate framework-8: a multifunctional nanoplatform for rechargeable in vivo persistent luminescence imaging and pH-responsive drug release, ACS Appl. Mater. Interfaces, 2018, 11, 1907-1916.

23 Z. Gong, et al., The synergistically improved afterglow and magnetic resonance imaging induced by $\mathrm{Gd}^{3+}$ doping in ZGGO: $\mathrm{Cr}^{3+}$ nanoparticles, Mater. Res. Bull., 2019, 113, 122-132.

24 L. Huang, et al., Near-Infrared Persistent Luminescence in a $\mathrm{Cr}^{3+}$-Doped Perovskite for Low-Irradiance Imaging, Chem. Mater., 2020, 32, 5579-5588.

25 R. S. King and D. A. Skros, Sunlight-activated near-infrared phosphorescence as a viable means of latent fingermark visualisation, Forensic Sci. Int., 2017, 276, e35-e39.

$26 \mathrm{~J}$. Li, et al., Specific recognition of breast cancer cells in vitro using near infrared-emitting long-persistence luminescent $\mathrm{Zn}_{3} \mathrm{Ga}_{2} \mathrm{Ge}_{2} \mathrm{O}_{10}: \mathrm{Cr}^{3+}$ nanoprobes, Nano-Micro Lett., 2015, 7, 138-145.

27 Y.-J. Li and X.-P. Yan, Synthesis of functionalized tripledoped zinc gallogermanate nanoparticles with superlong near-infrared persistent luminescence for long-term orally administrated bioimaging, Nanoscale, 2016, 8, 14965-14970.

28 J.-M. Liu, et al., Construction of molecularly imprinted nanoplatforms with persistent luminescence for the in vitro specific adsorption and in vivo targeted regulation of foodborne biotoxins, New J. Chem., 2019, 43, 15097-15104. 
29 J. Ren, et al., Novel Self-Activated Zinc Gallogermanate Phosphor: The Origin of its Photoluminescence, J. Am. Ceram. Soc., 2014, 97, 3197-3201, DOI: 10.1111/jace.13103.

30 F. Shen, C. Deng, X. Wang and C. Zhang, Effect of Cr on long-persistent luminescence of near-infrared phosphor $\mathrm{Zn}_{3} \mathrm{Ga}_{2} \mathrm{Ge}_{2} \mathrm{O}_{10}: \mathrm{Cr}^{3+}$, Mater. Lett., 2016, 178, 185-189.

31 Q. Wang, S. Zhang, Z. Li and Q. Zhu, Near Infrared-Emitting $\mathrm{Cr}^{3+} / \mathrm{Eu}^{3+}$ Co-doped Zinc Gallogermanate Persistence Luminescent Nanoparticles for Cell Imaging, Nanoscale Res. Lett., 2018, 13, 1-9.

32 T. Wang, et al., Tunable LLP via Energy Transfer between $\mathrm{Na}_{2-\mathrm{y}}\left(\mathrm{Zn}_{1-\mathrm{x}} \mathrm{Ga}_{\mathrm{x}}\right) \mathrm{GeO}_{4}$ Sosoloid Host and Emission Centers with the Assistance of $\mathrm{Zn}$ Vacancies, J. Phys. Chem. C, 2015, 119, 14047-14055.

$33 \mathrm{Y}$. $\mathrm{Wu}$, et al., Near-infrared long-persistent phosphor of $\mathrm{Zn}_{3} \mathrm{Ga}_{2} \mathrm{Ge}_{2} \mathrm{O}_{10}: \mathrm{Cr}^{3+}$ sintered in different atmosphere, Spectrochim. Acta, Part A, 2015, 151, 385-389.

$34 \mathrm{H}$. $\mathrm{Xu}$ and G. Chen, Enhancing near infrared persistent luminescence from $\mathrm{Cr}^{3+}$-activated zinc gallogermanate powders through $\mathrm{Ca}^{2+}$ doping, Opt. Mater. Express, 2017, 7, 2783-2792.

$35 \mathrm{X}$. Xu, et al., Broadly tunable emission from Mn-doped zinc gallogermanate phosphors through composition modification, Opt. Mater. Express, 2014, 4, 2433-2440.

36 Q. Zhu, et al., $\mathrm{Zn}_{3} \mathrm{Ga}_{2} \mathrm{Ge}_{2} \mathrm{O}_{10}: \mathrm{Cr}^{3+}$ Uniform Microspheres: Template-Free Synthesis, Tunable Bandgap/Trap Depth, and In Vivo Rechargeable Near-Infrared-Persistent Luminescence, ACS Appl. Bio Mater., 2018, 2, 577-587.

$37 \mathrm{~J}$. Yang, et al., Irradiation-free photodynamic therapy in vivo induced by enhanced deep red afterglow within NIR-I biowindow, Chem. Eng. J., 2020, 387, 124067.

38 Z.-H. Wang, et al., Bacterial biofilm bioinspired persistent luminescence nanoparticles with gut-oriented drug delivery for colorectal cancer imaging and chemotherapy, ACS Appl. Mater. Interfaces, 2019, 11, 36409-36419.

39 J.-M. Liu, D.-D. Zhang, G.-Z. Fang and S. Wang, Erythrocyte membrane bioinspired near-infrared persistent luminescence nanocarriers for in vivo long-circulating bioimaging and drug delivery, Biomaterials, 2018, 165, 39-47.

40 S. K. Gupta, B. Rajeshwari, S. N. Achary, A. K. Tyagi and R. M. Kadam, Controlling the luminescence in $\mathrm{K}_{2} \mathrm{Th}\left(\mathrm{PO}_{4}\right)_{2}: \mathrm{Eu}^{3+}$ by energy transfer and excitation photon: a multicolor emitting phosphor, New J. Chem. , 2020, 44, 14703-14711, DOI: 10.1039/ d0nj03117h.

41 S. K. Gupta, K. Sudarshan and R. Kadam, Tunable white light emitting $\mathrm{Sr}_{2} \mathrm{~V}_{2} \mathrm{O}_{7}: \mathrm{Bi}^{3+}$ phosphors: Role of bismuth ion, Mater. Des., 2017, 130, 208-214.

42 S. K. Gupta, et al., Lanthanide-doped lanthanum hafnate nanoparticles as multicolor phosphors for warm white lighting and scintillators, Chem. Eng. J., 2020, 379, 122314.

$43 \mathrm{H}$. Li, et al., Commendable $\mathrm{Pr}^{3+}$-activated $\mathrm{Ba}_{2} \mathrm{Ga}_{2} \mathrm{GeO}_{7}$ phosphor with high-brightness white long-persistent luminescence, J. Mater. Chem. C, 2019, 7, 6698-6705, DOI: 10.1039/c9tc01735f.
44 L. Lin, et al., A white long-lasting phosphor $\mathrm{Y}_{2} \mathrm{O}_{2} \mathrm{~S}: \mathrm{Tb}^{3+}$, $\mathrm{Sm}^{3+}$ : an improvement of $\mathrm{Y}_{2} \mathrm{O}_{2} \mathrm{~S}: \mathrm{Tb}^{3+}, \mathrm{J}$. Rare Earths, 2008, 26, 648-651, DOI: 10.1016/S1002-0721(08)60154-4.

45 L. Lin, Z. Zhao, W. Zhang, Z. Zheng and M. Yin, Photoluminescence properties and thermo-luminescence curve analysis of a new white long-lasting phosphor: $\mathrm{Ca}_{2} \mathrm{MgSi}_{2-}$ $\mathrm{O}_{7}: \mathrm{Dy}^{3+}$, J. Rare Earths, 2009, 27, 749-752, DOI: 10.1016/ S1002-0721(08)60328-2.

46 Y. Liu, B. Lei and C. Shi, Luminescent Properties of a White Afterglow Phosphor $\mathrm{CdSiO}_{3}: \mathrm{Dy}^{3+}$, Chem. Mater., 2005, 17, 2108-2113, DOI: 10.1021/cm0496422.

47 S. García-Dalí, et al., Activation of two-dimensional $\mathrm{MoS}_{2}$ nanosheets by wet-chemical sulfur vacancy engineering for the catalytic reduction of nitroarenes and organic dyes, Appl. Mater. Today, 2020, 20, 100678, DOI: 10.1016/j.apmt. 2020.100678 .

48 I. Matanovic, et al., Towards defect engineering in hexagonal $\mathrm{MoS}_{2}$ nanosheets for tuning hydrogen evolution and nitrogen reduction reactions, Appl. Mater. Today, 2020, 21, 100812, DOI: 10.1016/j.apmt.2020.100812.

49 Y.-M. Wang, et al., Optical-intensity modulator with InSb nanosheets, Appl. Mater. Today, 2020, 21, 100852, DOI: 10.1016/j.apmt.2020.100852.

50 S. Yan, et al., Zinc gallogermanate solid solution: A novel photocatalyst for efficiently converting $\mathrm{CO}_{2}$ into solar fuels, Adv. Funct. Mater., 2013, 23, 1839-1845.

51 S. K. Gupta, C. Hernandez, J. P. Zuniga, K. Lozano and Y. Mao, Luminescent PVDF nanocomposite films and fibers encapsulated with $\mathrm{La}_{2} \mathrm{Hf}_{2} \mathrm{O}_{7}: \mathrm{Eu}^{3+}$ nanoparticles, SN Appl. Sci., 2020, 2, 616, DOI: 10.1007/s42452-020-2412-1.

52 S. Chenu, et al., Tuneable Nanostructuring of Highly Transparent Zinc Gallogermanate Glasses and Glass-Ceramics, Adv. Opt. Mater., 2014, 2, 364-372, DOI: 10.1002/adom. 201400007.

53 S. Y. Bae, H. W. Seo, C. W. Na and J. Park, Synthesis of bluelight-emitting $\mathrm{ZnGa}_{2} \mathrm{O}_{4}$ nanowires using chemical vapor deposition, Chem. Commun., 2004, 1834-1835, DOI: 10.1039/ b405592f.

54 Q. Liu, et al., High-Yield Synthesis of Ultralong and Ultrathin $\mathrm{Zn}_{2} \mathrm{GeO}_{4}$ Nanoribbons toward Improved Photocatalytic Reduction of $\mathrm{CO}_{2}$ into Renewable Hydrocarbon Fuel, J. Am. Chem. Soc., 2010, 132, 14385-14387, DOI: 10.1021/ja1068596.

55 M. Wan, et al., Long afterglow properties of $\mathrm{Eu}^{2+} / \mathrm{Mn}^{2+}$ doped $\mathrm{Zn}_{2} \mathrm{GeO}_{4}$, J. Lumin., 2014, 145, 914-918, DOI: 10.1016/ j.jlumin.2013.09.011.

56 X. Hong, L. Zou, J. Zhao, C. Li and L. Cong, Dry-wet spinning of PVA fiber with high strength and high Young's modulus, MS\&E, 2018, 439, 042011.

57 J. K. Pandey, K. R. Reddy, A. P. Kumar and R. Singh, An overview on the degradability of polymer nanocomposites, Polym. Degrad. Stab., 2005, 88, 234-250. 